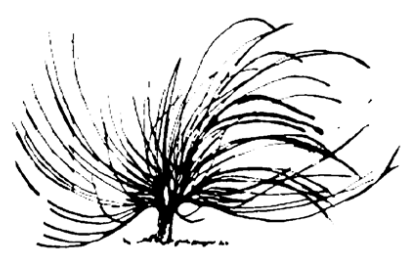

\title{
La "responsividad" en la escritura sobre las prácticas de enseñanza ${ }^{1}$
}

\author{
Márcia Cristina Greco Ohuschi ${ }^{2}$ \\ Universidad Federal de Pará \\ Brasil \\ marciaohuschi@yahoo.com.br
}

\begin{abstract}
Resumen
Este trabajo, vinculado al Proyecto de Investigación " $\mathrm{La}$ escritura sobre las prácticas de enseñanza en licenciaturas de Brasil, Costa Rica y Honduras: Registro, análisis y producción de conocimiento" (UFPA/CNPq), tiene como objetivo diagnosticar la "responsividad" manifestada en la escritura con respecto a las prácticas de enseñanza de profesorado en formación. A la luz de la lingüística aplicada, en la perspectiva socio-histórica del lenguaje, nos basamos en los presupuestos teóricos de Bakhtin/ Volochinov (1992) y Bakhtin (2003). En lo tocante a la noción bakhtiniana de "responsividad", nos apoyamos en el estudio de Menegassi (2008; 2009) y de Ohuschi (2013). Como la investigación aún está en curso, se presenta una muestra representativa, a partir de ejemplos de la capacidad de respuesta que se manifiesta en los informes de las prácticas realizadas por estudiantes de un grupo de Letras -Portugués- Universidad Federal de Pará (UFPA), Campus Castanhal. Los resultados revelan que el alumnado demuestra, al escribir acerca de la práctica docente, la capacidad de respuesta activa con expansión
\end{abstract}

http://dx.doi.org/10.15359/rep.esp-20-1.11

1 Este trabajo fue presentado en la Mesa Redonda "Escritura sobre las prácticas en la formación docente -retos de una investigación transnacional en Brasil, Costa Rica y Honduras", en el III Seminario de Investigación Educativa para Docentes y Estudiantes Universitarios, en la Universidad Pedagógica Nacional Francisco Morazán- Centro Universitario Regional de San Pedro Sula, en abril, 2015.

2 Profesora de la Universidad Federal de Pará. Doctora en Estudios del Lenguaje por la Universidad Estadual de Londrina. 
explicativa y ejemplificativa en niveles de explicación, comentario y crítica.

Palabras claves: Dialogismo, "responsividad", escritura, prácticas de enseñanza, profesorado en formación.

\begin{abstract}
This paper, which is linked to the research project $L a$ escritura sobre las prácticas de enseñanza en licenciaturas de Brasil, de Costa Rica y de Honduras: registro, análisis $y$ producción de conocimiento (UFPA/CNPq), aims to diagnose responsivity manifested in writing regarding pre-service teachers during their practicum. This research is situated within the Applied Linguistics field and is grounded on the socio-historical perspective of language, according to the theoretical assumptions of Bakhtin/ Volochinov (1992) and Bakhtin (2003). With regard to Bakhtin's notion of responsivity, we follow the studies of Menegassi (2008; 2009) and Ohuschi (2013). As research is still in progress, we present a representative sample of responsivity manifested in practicum reports of a writing class - Portuguese Language - at the Federal University of Pará (UFPA), Campus of Castanhal. The results show that trainees demonstrate, in writing about the teaching practicum, active responsivity with explanatory and illustrative expansion in levels of explanation, commentary, and criticism.
\end{abstract}

Keywords: dialogism, responsivity, writing, teaching practices, teachers in training

\title{
1. Consideraciones iniciales
}

$\mathrm{E}$ 1 proyecto de investigación "La escritura sobre las prácticas de enseñanza en licenciaturas de Brasil, de Costa Rica y de Honduras: registro, análisis y producción de conocimiento" (UFPA/CNPq) que se aprobó por el llamado universal 14/2014, incluye cinco universidades brasileñas situadas en cuatro regiones geográficas diferentes y dos universidades de países latinoamericanos vecinos, Costa Rica y Honduras. La asociación entre la UNA/Costa Rica, UPNFM/Honduras y varias universidades es un trabajo que se está 
haciendo, comprometido con el establecimiento de la investigación en las universidades y cualificación de su profesorado a través de su participación en las actividades de investigación.

El proyecto tiene como objetivo discutir el papel de la escritura en las carreras de grado y considerar que la escritura en las prácticas, para la formación del profesorado, no es solo una forma de documentar o presentar sus actividades, sino un trabajo que puede resultar en la producción de conocimiento. En este sentido, el proyecto se basa en tres aspectos: a) ¿cómo los textos escritos de las prácticas de enseñanza funcionan durante la grabación de una lección; b) ¿qué tipo de trabajo se puede realizar en el registro de una clase; y c) lo que se afirma en una clase basada en lo que estaba grabado. Por lo tanto, se analiza la escritura producida a partir de las prácticas de enseñanza como un hecho, como un objeto de procedimientos de análisis, además de la producción de conocimiento sobre la enseñanza.

Desde esta tendencia, en esta investigación tenemos la intención de enfatizar la capacidad de respuesta a partir de aspectos de la descripción de una clase en las prácticas de enseñanza en pregrado, en los textos escritos por sus estudiantes. Para ello, presentamos ejemplos de la capacidad de respuesta activa manifestada en informes de las prácticas, llamados "relatos reflexivos", producidos por alumnado de la carrera de Letras - Portugués, UFPA, campus de Castanhal, en el año de 2012.

\section{La "responsividad": aspectos teóricos}

Según Faraco (2009), después de los años 1925/1926, cuando la cuestión de la lengua cambió la dirección de los debates del Círculo de Bakhtin, esto fue consolidando progresivamente la gran metáfora del diálogo, que recogió sus reflexiones sobre lengua. Por lo tanto, la lengua se entiende a partir de su carácter socio-histórico-ideológico, como un fenómeno social de diálogo entre dos o más individuos.

El diálogo es, según Bakhtin/Volochinov (1992), una de las formas más importantes de la interacción verbal. En un sentido abarcador, se entiende como un amplio espacio de lucha entre las fuerzas socio-ideológicas entre voces sociales, con un verdadero enfrentamiento entre sus propias ideas y las de los otros sujetos, acuerdos, desacuerdos, refutaciones, preguntas, etc. Por lo tanto, no tiene sentido fuera de la diferencia, de la arena, de la interacción dialógica, por lo que no hay un discurso sin otros discursos (Sobral, 2009). Así, quien emite el mensaje 
se aclara como sujeto locutor y su discurso antes de decirlo, con el fin de establecer la imagen de la persona interlocutora a la que apela; existen, por lo tanto, actitudes de respuesta de ambas partes.

El sujeto receptor, que recibe y entiende la declaración (escrita u oral), adopta, para él, una actitud de respuesta que, según ha explicado Bakhtin (2003), se encuentra en desarrollo constante durante el proceso de comprensión. Asume una actitud de sujeto hablante, así que no es un mero oyente, participa activamente en el diálogo, ya que también establece la enunciación, puesto que la palabra se dirige a él.

De esta forma, la comprensión es una forma de diálogo, pues comprender es oponer una contestación a la palabra del sujeto locutor (Bakhtin y Volochinov, 1992), que muestra una respuesta activa de la enunciación. Con este sesgo, Menegassi (2009) define la capacidad de respuesta, señalando que no solo se deriva de las prácticas del lenguaje, sin embargo, estas son imprescindibles para que ocurran. De esta forma, es imprescindible comprender que la producción de un enunciado dirigido a la otra persona ya es, por sí, una probable respuesta a otros enunciados.

Según Bakhtin (2003), la comprensión de la respuesta puede manifestarse de tres maneras: activa, pasiva o silenciosa (efecto retardado). En el entendimiento de respuesta activa, el ente interlocutor, para entender la declaración, contesta de inmediato; expresa su posición, su juicio de valor, su acuerdo o desacuerdo; completa; etc. La comprensión pasiva es un elemento abstracto del hecho real (comprensión sensible activa) que se materializa en el acto real de respuesta y tiene en cuenta el cumplimiento de los plazos que se presentan en las interacciones. Menegassi (2009) explica que la pasividad de la respuesta se encuentra en el hecho de que la devolución al enunciado formulado por el sujeto locutor se manifiesta, en la otra persona, por la atención y cumplimiento de una petición, una solicitud o un orden. Esto registra la ocurrencia de la comprensión del enunciado por parte de quien oye, pero, simultáneamente, señala también una relación social altamente asimétrica, autoritaria en la relación entre hablante y oyente, ejemplificado por Bakhtin con el orden militar.

En la comprensión silenciosa (acción o retraso), la persona que llama tiene una respuesta, pero en otro momento, y puede estar en otro contexto, con otros sujetos interlocutores, además de que no siempre ocurre inmediatamente al enunciado. La comprensión puede ocurrir tarde o temprano, lo que fue escuchado y activamente atendido puede 
responderse en los discursos subsiguientes o en el comportamiento del público oyente (Bakhtin, 2003).

Nos damos de cuenta de que la comprensión (sea activa, pasiva o silenciosa) conduce al individuo a realizar una actitud receptiva, que también puede ser activa, pasiva o silenciosa. El hablante siempre espera una respuesta y no solo una repetición de su discurso.

La capacidad de respuesta (activa y silenciosa) se manifiesta cuando las palabras de otras personas se convierten en propias palabras, pues nuestro discurso está lleno de los demás discursos, de un grado variado de alteridad, perceptibilidad y relevancia. Estas palabras de los demás cargan su expresión, su tono valorativo que son asimilados y reelaborados (Bakhtin, 2003).

Además, en este proceso dialógico, la persona oradora/presentadora se convierte también en la otra de sí misma, porque todo sujeto hablante es por sí mismo un respondedor en mayor o menor grado, una vez que él no es el primer hablante, y presupone no solo la existencia del sistema de la lengua que utiliza, sino también de algunos enunciados anteriores. Así, "cada enunciado es un eslabón en la cadena complejamente organizada de otros enunciados [Cada enunciado é um elo na corrente complexamente organizada de outros enunciados]" (Bakhtin, 2003, p. 272).

En consecuencia, el sujeto locutor busca la virtualidad del sujeto interlocutor, con el fin de transferir a esta virtualidad y accionar los varios otros (interlocutores) que se internalizan en sí, transformando las palabras lejanas en sus propias palabras. En la producción de textos escritos, la persona locutora se mueve de papel de productora para el de interlocutora, al hacer inferencias, cubrir los vacíos dejados en el enunciado, etc. Es el movimiento inter-intra-inter-individual (Vygotsky, 1988), donde, intralocución, el locutor o locutora dialoga con su propio texto, por lo que es otra persona de sí misma.

Desde esta perspectiva, Menegassi (2009) señala que las diversas actitudes que responden a construir los enlaces de la corriente continua de la comunicación verbal en la que cada declaración individual es una respuesta a la relación anterior y, por lo tanto, implica una nueva actitud receptiva y así sucesivamente. El autor resume los principales aspectos de la capacidad de respuesta, caracterizando el dialogismo de Bakhtin, que sintetizamos: 
a. La responsividad es una exigencia de las prácticas sociales de interacción y un factor imprescindible para que las prácticas del lenguaje ocurran.

b. La propia formulación de un enunciado dirigido al otro ser constituye, por sí, una posible respuesta a otros enunciados que circulan en la sociedad.

c. El otro sujeto siempre ofrece una respuesta a la palabra de la que se apropia, generando un nuevo decir, marcado por las características de su nuevo rol locutor.

d. La respuesta a la palabra del otro sujeto produce mi palabra

e. El sujeto hablante siempre espera la respuesta de su público interlocutor, aguarda una activa comprensión responsiva.

f. "En la actitud responsiva pasiva, no se involucra necesariamente la verbalización de la respuesta. Su pasividad reside justamente en el hecho de que la devolución al enunciado formulado por el locutor se manifiesta, en el otro, por la atención y cumplimiento de un encargo, una solicitud o un orden. [Na atitude responsiva passiva, não se envolve necessariamente a verbalização da resposta. Sua passividade reside justamente no fato de que a devolutiva ao enunciado formulado pelo locutor se manifesta, no outro, pelo atendimento e cumprimento de um pedido, uma solicitação ou uma ordem]" (Menegassi, 2009, p. 169).

g. "La actitud responsiva silenciosa difiere de las anteriores por ser una materialización de efecto retardado a la respuesta inherente a un determinado enunciado. [A atitude responsiva silenciosa difere das anteriores por ser uma materialização de efeito retardado da resposta inerente a um determinado enunciado]" (Menegassi, 2009, p. 169).

De la "responsividad", el enunciado permite límites para establecer un diálogo, la adopción, por la declaración recibida, una actitud receptiva, como de venta, de acuerdo, en desacuerdo, la completa, la aplica, etc., ya que toda comprensión es activa (Bakhtin, 2003). Los límites de cada declaración se definen, según el pensador ruso, alternando los temas del discurso. Así, incluso antes de comenzar su discurso, hay diálogo con los discursos de otras personas, ya que "el hablante no es un Adán bíblico. [o falante não é um Adão bíblico]" (Bakhtin, 2003, p. 300), su discurso no es desconocido, se hace eco de los que le 
preceden. Para cerrarlo, debería haber diálogo con las declaraciones de respuesta de las demás personas, aunque sean en silencio. Por lo tanto, según Bakhtin (2003), el ser hablante termina su enunciado para dar lugar a su comprensión activamente responsiva, es decir, para pasarle la palabra al otro ser. Tomamos nota de que, puesto que el lenguaje tiene carácter socio-histórico-ideológico, este juego alternativo no está desprovisto de ideología, como sujetos sociales que interactúan generan su capacidad de respuesta a través de los valores sociales.

En virtud de este sesgo en el análisis de la "responsividad" en el discurso, a partir de textos escritos de géneros de correo electrónico y la entrevista, Menegassi (2008) presenta tres posibilidades para su caracterización: a) la capacidad de respuesta activa con la expansión explicativa e ilustrativa; b) la capacidad de respuesta pasiva sin expansión; c) la capacidad de respuesta activa sin expansión explicativa e ejemplificativa. Para facilitar la exposición, se presenta el marco elaborado por Ohuschi $(2013$, p. 48), que organiza las características planteadas por el autor:

Tabla 1: Sistematización de las características de la "responsividad"

\begin{tabular}{|c|c|c|}
\hline $\begin{array}{c}\text { "Responsividad" activa } \\
\text { con expansión explicativa } \\
\text { y ejemplificativa }\end{array}$ & $\begin{array}{c}\text { "Responsividad" } \\
\text { pasiva sin expansión }\end{array}$ & \begin{tabular}{|c|} 
"Responsividad" activa \\
sin expansión explicativa \\
y ejemplificativa
\end{tabular} \\
\hline $\begin{array}{l}\text { Pasa, primero, por la } \\
\text { comprensión silenciosa, } \\
\text { a continuación, por la } \\
\text { comprensión de efecto } \\
\text { retardado, por fin, se } \\
\text { manifiesta activa y } \\
\text { reflexivamente. }\end{array}$ & $\begin{array}{l}\text { Se produce una } \\
\text { respuesta directa, } \\
\text { sin desplazamiento } \\
\text { temporal. }\end{array}$ & $\begin{array}{l}\text { Ocurre una actitud } \\
\text { responsiva objetiva. }\end{array}$ \\
\hline $\begin{array}{l}\text { La manifestación ocurre } \\
\text { en un discurso posterior, } \\
\text { hay un desplazamiento } \\
\text { temporal. }\end{array}$ & $\begin{array}{l}\text { No permite la } \\
\text { continuación del } \\
\text { diálogo. }\end{array}$ & $\begin{array}{l}\text { Las reflexiones del } \\
\text { sujeto productor no son } \\
\text { personales, sino propias de } \\
\text { lo que se le pidió. }\end{array}$ \\
\hline $\begin{array}{l}\text { Se producen reflexiones } \\
\text { personales. } \\
\text { Permite que el diálogo } \\
\text { continúe. }\end{array}$ & & $\begin{array}{l}\text { Permite la continuación } \\
\text { del diálogo, pero de forma } \\
\text { conducida. }\end{array}$ \\
\hline
\end{tabular}


Sobre la base de las categorías bakhtinianas de la capacidad de respuesta, expandido en Menegassi (2008), se propone la Tabla 2 (Ohuschi, 2013, p. 161-162), con extensiones de diferentes subcategorías propuestas por el autor. Fueron ampliadas en función del contexto y los datos generados por la encuesta, a partir de las actitudes de respuesta de los grupos docentes.

Tabla 2: Niveles de "responsividad" en los diálogos de los profesores

\begin{tabular}{|c|c|c|}
\hline \begin{tabular}{|l|} 
CATEGORÍAS \\
\end{tabular} & NIVELES & SIGNIFICADO \\
\hline \multirow{7}{*}{$\begin{array}{l}\text { "Responsividad" } \\
\text { activa con } \\
\text { expansión } \\
\text { explicativa y } \\
\text { ejemplificativa }\end{array}$} & Crítica & $\begin{array}{l}\text { Respuesta inmediata del sujeto socio de la } \\
\text { situación de interacción, con análisis, juicio y } \\
\text { evaluación. }\end{array}$ \\
\hline & Opinión & $\begin{array}{l}\text { Respuesta inmediata, normalmente ligada a la } \\
\text { crítica, a partir del posicionamiento, revela marcas } \\
\text { de reflexión personal. }\end{array}$ \\
\hline & $\begin{array}{l}\text { Comentario y } \\
\text { ejemplificación }\end{array}$ & $\begin{array}{l}\text { Respuesta inmediata que puede presentarse: a) por } \\
\text { medio de las vivencias personales; b) a partir de las } \\
\text { experiencias del trabajo cotidiano de aula -saber } \\
\text { experiencial- (Tardif, 2002); c) por las experiencias } \\
\text { de la graduación -saber profesional- (Tardif, 2002). }\end{array}$ \\
\hline & Explicación & $\begin{array}{l}\text { Respuesta inmediata del sujeto socio de la } \\
\text { situación de interacción, con marcas de reflexión, } \\
\text { explicita comprensión activa. }\end{array}$ \\
\hline & Desacuerdo & $\begin{array}{l}\text { Respuesta inmediata del sujeto socio de la } \\
\text { situación de interacción, discrepa de lo que está } \\
\text { siendo discutido. }\end{array}$ \\
\hline & Sugerencia & $\begin{array}{l}\text { Respuesta inmediata, evidenciada a partir de la } \\
\text { búsqueda de solución de un problema encontrado, } \\
\text { demuestra madurez. }\end{array}$ \\
\hline & Cuestionario & $\begin{array}{l}\text { Respuesta inmediata, movilizada por medio de una } \\
\text { pregunta, con una marca explícita de continuidad } \\
\text { del diálogo. }\end{array}$ \\
\hline $\begin{array}{l}\text { "Responsividad" } \\
\text { activa sin } \\
\text { expansión } \\
\text { explicativa y } \\
\text { ejemplificativa }\end{array}$ & Acuerdo & $\begin{array}{l}\text { Respuesta inmediata en que el sujeto socio de la } \\
\text { situación de interacción demuestra estar de acuerdo } \\
\text { con lo que está siendo debatido, solo para que la } \\
\text { discusión perdure, sin marcas de reflexión personal. }\end{array}$ \\
\hline $\begin{array}{l}\text { "Responsividad" } \\
\text { pasiva sin } \\
\text { expansión }\end{array}$ & Leve & $\begin{array}{l}\text { El sujeto socio no realiza las tareas solicitadas, } \\
\text { imposibilita la instauración del diálogo. }\end{array}$ \\
\hline
\end{tabular}




\begin{tabular}{|l|l|l|}
\hline $\begin{array}{l}\text { "Responsividad" } \\
\text { silenciosa }\end{array}$ & Duda & $\begin{array}{l}\text { El sujeto socio demuestra aún tener duda o } \\
\text { dificultad sobre algo discutido, por presentarse en } \\
\text { proceso de sedimentación del conocimiento. }\end{array}$ \\
\cline { 2 - 3 } & Comprensión & $\begin{array}{l}\text { El sujeto socio revela intentos de comprensión, se } \\
\text { encuentra en proceso de internalización (Vygotsky, } \\
1988) .\end{array}$ \\
\hline
\end{tabular}

\section{La responsividad activa en relatos reflexivos}

En esta sección, presentamos ejemplos de la capacidad de respuesta activa en la escritura de estudiantes del área de letras en las prácticas de enseñanza, a través de la producción de relatos reflexivos, solicitados en la asignatura Etapa Supervisada en la Enseñanza Primaria, en 2012. Traducimos los datos para el castellano y presentamos en las notas al pie, la forma en que cada estudiante hizo la escritura en portugués.

Los informes se producen en parejas, que llevaron a cabo los pasos de observación (10 horas de clase) y práctica docente (6 horas de clase) de portugués, idioma de prácticas en la educación primaria. Los ejemplos fueron sacados de siete informes dobles identificados como pareja y el número correspondiente (uno a siete).

Los ejemplos seleccionados pretenden destacar algunas características de la etapa, lo que demuestra su contribución a la educación de los maestros y las maestras.

Estas características se han basado en Pimenta y Gonçalves (en Pimenta, 2002, p. 13-14), que afirman sobre la etapa lo siguiente:

Tendrá por finalidad propiciar al alumno una aproximación a la realidad en la que actuará. Por lo tanto, no se debe poner la etapa como el polo práctico del curso, sino como una aproximación a la práctica, en la medida en que será consecuente a la teoría estudiada en el curso, que, a su vez, deberá constituirse en una reflexión sobre y desde la realidad de la escuela pública. ...Es necesario que se asuma que la actividad ocurrirá, efectivamente, en el momento en que el alumno sea profesor, en la práctica. Es decir, un curso no es la práctica docente, pero es la teoría sobre la práctica docente y será tan más formador a medida que las asignaturas todas tengan como eje la realidad escolar brasileña. [Terá por finalidade propiciar ao aluno uma aproximação à realidade 
na qual irá atuar. Portanto não se deve colocar o estágio como o pólo prático do curso, mas como uma aproximação à prática, na medida em que será conseqüente à teoria estudada no curso, que, por sua vez, deverá se constituir numa reflexão sobre e a partir da realidade da escola pública (...). É preciso que se assuma que a atividade ocorrerá, efetivamente, no momento em que o aluno for professor, na prática. Ou seja, um curso não é a prática docente, mas é a teoria sobre a prática docente e será tão mais formador à medida que as disciplinas todas tiverem como ponto de partida a realidade escolar brasileira].

Por lo tanto, delimitamos cuatro aspectos, de los cuales buscamos diagnosticar la capacidad de respuesta activa del alumnado: a) las prácticas teorizan sobre la práctica docente; $b$ ) la etapa a menudo es la primera experiencia en la enseñanza del personal académico; c) las prácticas ofrecen una reflexión sobre la realidad de la escuela pública; d) la fase proporciona la autorreflexión.

\subsection{Las prácticas teorizan sobre la práctica docente}

En los extractos que ilustran el punto de que las prácticas están teorizando sobre la práctica docente (Pimenta; Gonçalves en Pimenta, 2002), confirmamos la hipótesis - planteada por el proyecto de investigación "La escritura sobre las prácticas de enseñanza en licenciaturas de Brasil, de Costa Rica y de Honduras: registro, análisis y producción de conocimiento" (UFPA/CNPq) - en que el alumnado, para analizar situaciones vividas en las prácticas de enseñanza, tiende a comparar una clase particular celebrada con los procedimientos defendidos por un autor o autora o corriente teórica.

$\mathrm{Al}$ observar esta premisa, consideramos que, en los tres primeros ejemplos, la "responsividad" mostrada por los estudios para demostrar la comprensión activa de las teorías de la enseñanza y el aprendizaje LP incautada en el curso, en especial de las asignaturas "Ensino-aprendizagem I" y "Ensino-aprendizagem II", que son anteriores a el "Estágio Supervisionado no Ensino Fundamental".

En este sentido, en los siguientes ejemplos, se observó que el alumnado demuestran comprensión activa de las teorías de la enseñanza y el aprendizaje LP adquiridas en el curso, lo que muestra que las prácticas están teorizando sobre la práctica docente (Pimenta y Gonçalves 
en Pimenta, 2002). La pareja 1 muestra las teorías de conceptos de escritura, la pareja 2 sobre los tipos de preguntas de lectura, la pareja 3 demuestra la comprensión de los conceptos gramaticales y la pareja 4 de la interacción.

Por lo tanto, las declaraciones escritas presentan la respuesta inmediata, a través de experiencias durante las prácticas supervisadas, con marcas de reflexión, que señalan la comprensión activa de crítica (Ohuschi, 2013), como se indica a continuación:

En la primera clase observada $\left(6^{\circ} 03\right)$, percibimos que la primera actividad ... traía, según Sercundes (1997), la concepción de escritura como un don, pues la solicitud de producción de texto cobrado por la profesora no tenía una preocupación de trabajar antes con los alumnos, para así, pedir la producción escrita, seguida por el trabajo con la reescritura $^{3}$ (pareja 1).

La pareja 1 muestra la visión metodológica de Sercundes (1997), que cuenta con tres conceptos de escritura: como un don, como consecuencia y como trabajo. La pareja 1 identifica en la clase observada, el concepto de la escritura como un regalo, ya que no hay actividades anteriores de producción textual.

Aunque el grupo académico no presenta datos más concretos para probar su afirmación, como el comando de producción textual, por ejemplo, demuestra la comprensión activa de la perspectiva estudiada durante la disciplina "Ensino-aprendizagem do Português II". Por lo tanto, observamos la manifestación de la "responsividad" activa con la expansión explicativa e ilustrativa en el nivel de explicación porque, como Ohuschi (2013, p. 161), hay una "respuesta inmediata del compañero a la situación de interacción, con marcas de reflexión", que explicita la comprensión activa.

En la reflexión que hicimos respecto a la segunda clase $\left(8^{\mathrm{a}} 1\right)$, vimos el trabajo con el género Jartum y a partir de él había algunas cuestiones interpretativas. Nos sentimos contentos al percibir que la actividad de un libro didáctico, traída por la profesora, tenía una propuesta que se aparta del método tradicional. En la

3 Na primeira turma observada $\left(6^{\circ} 03\right)$, percebemos que a primeira atividade ... trazia, segundo Sercundes (1997), a concepção de escrita como um dom, pois a solicitação de produção de texto cobrado pela professora não tinha uma preocupação de se trabalhar antes com os alunos, para assim, pedir a produção escrita, seguida pelo trabalho com a reescrita. 
tarea propuesta, había algunas cuestiones clasificadas por Menegassi (2010a) como preguntas de respuestas interpretativas. Por medio de ellas, el alumno-lector reflexiona sobre lo que está leyendo y después logra desarrollar su capacidad crítica, considerando en las respuestas sus vivencias personales ${ }^{4}$ (pareja 2).

La pareja 2 también muestra la capacidad de respuesta activa de explicación, mediante la exposición de la observación de la clase, los tipos de preguntas de lectura que la maestra estaba trabajando, a través del libro de texto, aunque no los presentan textualmente. En el curso "Ensino-aprendizagem do Português II", el alumnado estudió las teorías de lectura, derivadas de la psicolingüística y la lingüística aplicada, y se analizaron cuestiones presentes en los libros de texto. De esta manera, traen el conocimiento interiorizado y tratan de vincular la teoría con la práctica. En este ejemplo, se hace referencia específicamente a un texto de Menegassi (2010), que clasifica las preguntas de lectura: preguntas de respuesta literal, inferencial e interpretativa.

Además, podemos ver en esta declaración, la manifestación de la capacidad de respuesta activa con expansión de comentario (Ohuschi, 2013), en "Nos alegró constatar que la actividad de un libro de texto, traído por el profesor, tenía una propuesta que se apartaba del método tradicional". Los estudiantes demuestran una respuesta inmediata que surge de las experiencias de grado -relacionadas con el conocimiento profesional- (Tardif, 2002).

Todavía existe una posición tradicionalista por parte de los profesores, en que la enseñanza de la gramática normativa, aquella que se basa en el estudio de las normas gramaticales, sigue siendo predominante, como en el caso de la profesora G., que utilizó el texto como pretexto. La gramática descriptiva también es utilizada, por medio de conceptos acabados sacados de libros didácticos,presente en la observación de las clases de la profesora

4 Na reflexão que fizermos acerca da segunda turma ( $\left.8^{\mathrm{a}} 1\right)$, vimos o trabalho com o gênero Cartum e a partir dele havia algumas questões interpretativas. Ficamos contentes por notar que a atividade de um livro didático, trazida pela professora, tinha uma proposta que se afastava do método tradicional. Na tarefa proposta, havia algumas questões classificadas por Menegassi (2010a) como perguntas de respostas interpretativas. Por meio delas, o aluno-leitor reflete sobre o que está lendo e depois consegue desenvolver sua capacidade crítica, considerando nas respostas suas vivências pessoais. 
N., en que ella solicitaba a los alumnos que saquen conceptos listos del libro didáctico para apenas reproducir en los cuadernos ${ }^{5}$ (pareja 3).

La pareja 3 muestra la comprensión activa sobre los conceptos gramaticales, también estudió en la asignatura "Ensino-aprendizagem do Português II", a través de Travaglia (1996), que trata sobre tres concepciones de gramática: normativa, descriptiva e internalizada. En el extracto, la pareja identifica los dos primeros, relacionándolos con una postura tradicionalista que, en el primer caso, la profesora utiliza el texto como pretexto para enseñar reglas de la gramática $\mathrm{y}$, en el segundo, pidió una copia de conceptos gramaticales (que entiende cómo descripción de la lengua) dispuestos en el libro de texto.

Tomamos nota de que, aunque de forma simplificada, los grupos practicantes tratan de relacionar la práctica experimentada con lo que han estudiado en la teoría (Pimenta y Gonçalves, en Pimenta, 2002) y, en las prácticas están teorizando sobre la práctica docente. Por lo tanto, caracterizan la capacidad de respuesta activa con expansión de explicación (Ohuschi, 2013), dado que tratan de explicar la práctica de la enseñanza a través de las teorías internalizadas.

Sin embargo, cabe destacar que es notoria la prevalencia, acerca de las lecciones observadas, de metodologías tradicionales de enseñanza, lo que resulta probablemente de una visión académica para observar las clases de las demás personas. Esto no ocurre cuando habla de su propia práctica, como se muestra en el siguiente ejemplo:

A partir de las clases impartidas durante la práctica docente, percibimos la importancia de promover la interacción entre profesor y alumnos, teniendo en cuenta acontecimientos de lo cotidiano. Así, la clase se vuelve más dinámica, productiva y los educandos pasan a ser más reflexivos y cuestionadores ante la sociedad. En ese sentido, Bakhtin/Volochinov (2004) dicen que el lenguaje es el resultado del intercambio de influencia del

5 Há ainda uma postura tradicionalista por parte dos professores, em que o ensino da gramática normativa, aquela que se baseia no estudo das normas gramaticais, ainda é predominante, como no caso da professora G., que utilizou o texto como pretexto. A gramática descritiva também é utilizada, por meio de conceitos prontos retirados de livros didáticos, presente na observação das aulas da professora N., em que ela pedia para os alunos retirarem conceitos prontos do livro didático para apenas copiar nos cadernos. 
pensamiento de un individuo sobre el otro. Por eso, entendemos que instigamos la participación de los discursos y buscamos (todo el tiempo) promover la interacción en clase ${ }^{6}$ (Pareja 4).

La diferencia de anteriores declaraciones, en las que los practicantes hacen referencia a las lecciones observadas, considerándolas, en su mayoría "tradicionales", en este ejemplo, hay una muestra de escritura acerca de la pasantía. En este contexto, tratan de demostrar que la interacción promovida entre docentes y estudiantes hace que la clase sea "más dinámica y productiva", y asegura que el alumnado sea "más reflexivo y cuestionador ante la sociedad". De ese modo, la pareja cree que su clase era "interaccionista", a diferencia de las clases observadas, consideradas "tradicionales".

Promover la interacción docente-estudiante es un factor importante, pero no garantiza que la clase se base en la concepción interaccionista de lengua. Es necesario que se trabajen las prácticas del lenguaje (lectura, escritura y análisis lingüístico) para promover la interacción del estudiantado con el texto y el lenguaje. Por lo tanto, diagnosticamos la "responsividad" silenciosa de la comprensión, en que la pareja demuestra un intento de comprensión de la teoría; pero, permanece, todavía, en proceso de internalización (Ohuschi, 2013).

\subsection{La etapa a menudo es la primera experiencia en la enseñanza del personal docente}

En los siguientes extractos, confirmamos que, normalmente, las prácticas son la primera experiencia en la enseñanza del alumnado. Las declaraciones de la pareja 1 y la pareja 5 demuestran este hecho y responden activamente, con comentarios, a través de las experiencias de las prácticas.

Basándose en lo expuesto anteriormente, podemos concluir que la asignatura 'Etapa supervisada en la enseñanza primaria ' fue de

6 A partir das aulas ministradas durante o estágio de regência, percebemos a importância de se promover a interação entre professor e alunos, levando em consideração acontecimentos do cotidiano. Assim, a aula torna-se mais dinâmica, produtiva e os educandos passam a ser mais reflexivos e questionadores diante da sociedade. Nesse sentido, Bakhtin/Volochinov (2004) dizem que a linguagem é o resultado da troca de influência do pensamento de um indivíduo sobre o outro. Por isso, entendemos que instigamos a participação dos discentes e procuramos (o tempo todo) promover a interação em sala. 
gran importancia, pues nos permitió el contacto con un universo hasta entonces desconocido, pues aún no habíamos tenido la oportunidad de tener un contacto directo y una relación profesor-alumno, en la condición de docentes. Este posicionamiento nos hizo reflexionar mucho sobre nuestra futura profesión, una vez que nos encontramos con una realidad que no es fácil, y que a veces sólo la fuerza de voluntad no es suficiente $^{7}$ (pareja 1)

Mostrando que la práctica fue el primer contacto de pareja con el ambiente escolar, en la condición de docentes, comentaron que esta experiencia les llevó a reflexionar sobre su futura profesión. Por lo general, esta primera experiencia es decisiva para la gran mayoría del estudiantado, que decide, en ese momento, si quiere ser profesor o profesora. Es característica, en el extracto, la capacidad de respuesta activa con expansión en nivel de comentario, ya que la respuesta inmediata se presenta a través de las experiencias personales de aprendices en la escuela (Tardif, 2002).

... La práctica docente fue de extrema importancia, para acompañar el trabajo de un profesor con experiencia escolar y graduado. ... La etapa de la enseñanza fundamental proporcionó vivir con el alumnado y aprender cómo es la realidad dentro del aula. También somos conscientes de los defectos y deficiencias educativas que no se pueden cometer en el futuro, que sirve como la base de nuestra propia experiencia como futuros profesores de portugués $^{8}$ (pareja 5).

7 Com base no que foi exposto anteriormente, podemos concluir que a disciplina 'Estágio supervisionado no ensino fundamental' foi de grande importância, pois nos permitiu entrar em contato com um universo até então desconhecido, pois ainda não tínhamos tido a oportunidade de ter um contato direto e uma relação professor-aluno, na condição de docentes. Esse posicionamento nos fez refletir muito sobre nossa futura profissão, uma vez que nos deparamos com uma realidade que não é fácil, e que às vezes, somente a força de vontade não é o suficiente.

8 ... O estágio foi de extrema importância, para acompanharmos o trabalho de um professor com experiência escolar e formado. ... O estágio do ensino fundamental proporcionou vivermos com o alunado e aprendermos como é a realidade dentro da sala de aula. Também percebemos as faltas e deficiências de ensino que não podemos cometer no futuro, servindo como base para a nossa própria vivência como futuros professores de Português. 
La pareja 5 muestra la importancia de lograr constituirse en personal docente capacitado en el ejercicio de su profesión, también se manifiesta la capacidad de respuesta activa de comentario, por medio de vivencias en la escuela, como se señala en "nos permitió vivir con los estudiantes". Al final de la enunciación, la pareja menciona fallas y deficiencias que no quieren cometer en el futuro, probablemente refiriéndose a la visión, que ya se ha dicho, de que el personal docente observado comete fallos por no seguir cierta teoría, como si fuera ese un factor negativo.

\subsection{Las prácticas ofrecen una reflexión sobre la reali- dad de la escuela pública}

En el conjunto de 2 parejas, 6 y 7, se observa la reflexión sobre las realidades de la escuela pública (Pimenta; Gonçalves en Pimenta, 2002), acercándose a la realidad en la cual en el futuro van a trabajar. $\mathrm{Al}$ escribir sobre este aspecto, se caracteriza la capacidad de respuesta activa expansiva de la crítica, en la que hay una respuesta inmediata con el análisis, el juicio y la evaluación (OHUSCHI, 2013).

... la profesora pidió solamente que los alumnos reprodujeran del libro didáctico el asunto y el respectivo ejercicio de la clase. ... Percibimos que esa didáctica ocurrió porque la profesora tuvo que ministrar clases en los dos grupos al mismo tiempo, y la solución encontrada por ella fue esa ... ¿Nos preguntamos por qué el profesor se somete a un método tan lamentable como este?? (Pareja 2).

Los equipos practicantes experimentan una situación que a menudo ocurre en las escuelas públicas de Brasil, donde el profesorado tiene que impartir dos clases al mismo tiempo, para cubrir la falta de otro maestro o maestra. Con esto, normalmente, deja en una de las clases una actividad con el único objetivo de "rellenar la clase", en este caso, la copia del contenido y el ejercicio del libro de texto. El equipo

9 ... a professora pediu somente que os alunos copiassem do livro didático o assunto e o respectivo exercício da aula. ... Percebemos que essa didática aconteceu porque a professora teve que ministrar aulas nas duas turmas ao mesmo tempo, e a solução encontrada por ela foi essa ...Perguntamo-nos por que o professor se submete a um método tão lastimável como esse? 
académico evalúa este procedimiento como un "método tan lamentable", con lo cual muestra "responsividad" activa expansiva de la crítica.

Hemos percibido una gran falta de preparación de los profesores de lengua materna. Las clases que hemos observado presentaban señales de que no fueron proyectadas y sí que ya venían siendo reproducidas hace tiempo, lo que nos revela la comodidad de los profesores en la elaboración de su plan de enseñanza ${ }^{10}$ (pareja 6).

La pareja 6 reflexiona sobre otro problema que se encuentra en las escuelas públicas: la falta de planificación de las lecciones. Sin embargo, vale la pena señalar que este hecho no significa necesariamente mostrar "falta de preparación de los maestros", como destacaron, visto que, en Brasil, una serie de factores, tales como el exceso de trabajo, los bajos sueldos, la falta de tiempo, además de la clase para preparar, materiales y corregir exámenes y actividades, puede contribuir para este procedimiento. Así, esta pareja se manifiesta acerca de la capacidad de respuesta activa expansiva de crítica contra una realidad; sin embargo, no ubica los factores externos que pueden causar tal práctica.

Lo que llamó mucha atención en la observación de la" clase de repetidores ", en especial, la demostración de un extremo prejuicio que la clase viene sufriendo, como si hubieran escogido en todas las clases los" peores "alumnos, agregándoles en un mismo grupo. Además, la sala en que esta clase estaba alojada no proporcionaba el mínimo de confort, por la falta de espacio físico, la falta de sillas y mesa, hasta para la propia profesora ... ${ }^{11}$ (Pareja 7).

La pareja 7 muestra "responsividad" activa de crítica al evaluar dos situaciones de la realidad de las escuelas públicas brasileñas. La primera, observar el perjuicio sufrido por un grupo ya calificado como

10 Percebemos um grande despreparo dos professores de língua materna. As aulas que observamos pareciam que não foram planejadas e sim que já vinham sendo reproduzidas há bastante tempo, o que nos revela a comodidade dos professores na elaboração de seu plano de docência.

11 O que chamou muita atenção na observação da "turma de repetentes", em especial, a demonstração de um extremo preconceito que a classe vem sofrendo, como se tivessem escolhido em todas as turmas os "piores" alunos, lotando-os juntos. Além disso, a sala em que essa turma estava alojada não fornecia o mínimo de conforto, pela falta de espaço físico, falta de carteiras e mesa, até para a própria professora ... 
"clase de repetidores" y, en la segunda, las malas condiciones de la estructura física de las escuelas, haciendo hincapié en la falta de mesa incluso para la profesora.

\subsection{La fase proporciona la autorreflexión}

En el siguiente fragmento, se observa la autorreflexión de la pareja 1 de su etapa de pasantía al manifestar una "responsividad" activa expansiva de la crítica, a través del análisis, el juicio y la evaluación (OHUSCHI, 2013).

La sexta clase [de la regencia] fue de suma importancia no sólo para los alumnos que reflexionaron sobre sus propias respuestas, sino también por parte de los pasantes que, con las respuestas de los alumnos, pudieron reflexionar sobre las preguntas formuladas y las diversas respuestas que surgieron y que aún no habían sido pensadas ${ }^{12}$ (pareja 1 ).

La autorreflexión de la pareja 1 fue confirmada en las preguntas de lectura preparadas para la clase, ya que sus respuestas superaron sus expectativas, por presentar otras posibilidades. Vale la pena señalar que, en el corpus seleccionado, solamente esta pareja demostró autorreflexión. Este aspecto muestra que la mayoría de no reflexionan acerca de su desempeño docente en la práctica. Inferimos que esto se debe al hecho de que normalmente no reflexionan acerca de los equívocos para no explicitar sus propios errores.

A partir del análisis, con el fin de sistematizar los resultados obtenidos, presentamos la Tabla 3, que expone los niveles de "responsividad" diagnosticados en cada aspecto observado.

12 A sexta aula [da regência] foi de suma importância não só para os alunos que refletiram sobre suas próprias respostas, mas também por parte dos estagiários que, com as respostas dos alunos, puderam refletir sobre as perguntas formuladas e as diversas respostas que surgiram e que ainda não haviam sido pensadas. 
Tabla 3: sistematización de los resultados

\begin{tabular}{|c|c|}
\hline ASPECTO OBSERVADO & NIVEL DE "RESPONSIVIDAD" \\
\hline $\begin{array}{l}\text { La práctica teoriza sobre la práctica } \\
\text { docente }\end{array}$ & $\begin{array}{l}\text { - "Responsividad" activa con la } \\
\text { expansión explicativa e ilustrativa - } \\
\text { nivel de explicación } \\
\text { - "Responsividad" pasiva -nivel de } \\
\text { comprensión }\end{array}$ \\
\hline $\begin{array}{l}\text { La etapa a menudo es la primera } \\
\text { experiencia en la enseñanza de la } \\
\text { academia }\end{array}$ & $\begin{array}{l}\text { - "Responsividad" activa con la } \\
\text { expansión explicativa e ilustrativa - } \\
\text { nivel de comentario }\end{array}$ \\
\hline $\begin{array}{l}\text { Las prácticas ofrecen una reflexión } \\
\text { sobre la realidad de la escuela } \\
\text { pública }\end{array}$ & $\begin{array}{l}\text { - "Responsividad" activa con la } \\
\text { expansión explicativa e ilustrativa - } \\
\text { nivel de crítica }\end{array}$ \\
\hline La fase proporciona la autorreflexión & $\begin{array}{l}\text { - "Responsividad" activa con la } \\
\text { expansión explicativa e ilustrativa - } \\
\text { nivel de crítica }\end{array}$ \\
\hline
\end{tabular}

\section{Consideraciones finales}

En este trabajo, presentamos, como muestra representativa, ejemplos de la capacidad de respuesta activa manifiesta en informes de práctica. En ella demostramos el gran aporte de las prácticas en la formación inicial del profesorado y enfocamos los aspectos de la descripción de una clase que se requieren y efectivamente se encontraron en textos escritos de estudiantes de la carrera de Letras - Portugués, UFPA - Campus Castanhal.

Los ejemplos de la etapa acerca de la teorización sobre la práctica docente demuestran: a)la capacidad de respuesta activa expansiva de explicación, en la que el alumnado expone la comprensión activa de las teorías de la enseñanza y el aprendizaje del proceso de lengua portuguesa; b) la "responsividad" silenciosa de comprensión, en que el alumnado muestra tentativas de comprensión de las teorías estudiadas, por cumplir el proceso de interiorización del conocimiento (Vygostky, 1988). En los ejemplos de esa etapa, a menudo, se evidencia que esa es la primera experiencia en la enseñanza académica, en la cual se caracteriza la capacidad de respuesta activa expansiva del comentario a través de las experiencias adquiridas. Los ejemplos que la etapa proporciona para la reflexión sobre la realidad de las escuelas públicas destacan la "responsividad" activa expansiva de la crítica, en la que hay una 
respuesta inmediata, con el análisis, el juicio y la evaluación, así como el ejemplo de la etapa que proporciona la autorreflexión.

De ese modo, se espera que los resultados ayuden a desarrollar el conocimiento actual en lo que se refiere a las formas de escritura involucradas en la formación para la docencia, y que permitan comparar procedimientos adoptados en diferentes áreas de formación e instituciones y sirvan como fundamento para apuntar acciones didácticas y reformulaciones curriculares que contribuyan al desarrollo de las licenciaturas, en este caso, de la Licenciatura en Letras-Portugués. 


\section{Referencias}

Bakhtin, M; Volochinov, V. N. (1992). Marxismo e filosofia da linguagem. Hucitec.

Bakhtin, M. (2003). Estética da criação verbal. Martins Fontes.

Faraco, C. A. (2009). Linguagem \& Diálogo: as idéias linguísticas do Círculo de Bakhtin. Parábola Editorial.

Menegassi, R. J. (2008). Responsividade e dialogismo no discurso escrito. En P. NAVARRO (Eds.), O discurso nos domínios da linguagem e da história (pp.135-148). Claraluz.

Menegassi, R. J. (2009, $1^{\text {er }}$ semestre). Aspectos da responsividade na interação verbal. Revista Língua \& Letras. http://e-revista.unioeste.br/index.php/linguaseletras/article/view/2257

Menegassi, R. J. (2010), Perguntas de leitura. En R. J. Menegassi (Ed.), Leitura e ensino (pp. 167-190). Eduem.

Pimenta, S. G. (2002). O estágio na formação de professores: Unidade teoria e prática? Cortez.

Ohuschi, Márcia Cristina Greco. (2013). Ressignificação de saberes na formação continuada: a responsividade docente no estudo das marcas linguístico-enunciativas dos gêneros notícia e reportagem [Tesis de doutoramento no publicada]. Universidade Estadual de Londrina, Londrina.

Sercundes, M. M. I. (1997). Ensinando a escrever. En J. W.Geraldi, B. Citelli (Eds.), Aprender e ensinar com textos dos alunos (pp. 7597). Cortez.

Sobral, A. (2009). Do dialogismo ao gênero: as bases do pensamento do Círculo de Bakhtin. Mercado de Letras.

Tardif, M. (2002). Saberes Docentes e Formação Profissional. Vozes.

Travaglia, L. C. (1996). Gramática e interação: uma proposta para o ensino de gramática no $1^{\circ}$ e $2^{\circ}$ graus. Cortez.

Vygotsky, L. S. (1988). A formação social da mente (2. $\left.{ }^{\text {ed }}\right)$. Martins Fontes. 
